Adv. Studies Theor. Phys., Vol. 4, 2010, no. 13, 615 - 632

\title{
Canonical Exterior Formalism on Group Manifold for (1+1)-Dimensional Supergravity
}

\author{
O. S. Zandron ${ }^{1}$ \\ Facultad de Ciencias Exactas Ingeniería y Agrimensura de la UNR \\ Av. Pellegrini 250, Facultad de Química \\ Ingeniería en Tecnología de los Alimentos UCEL \\ Av. Pellegrini 1332 - 2000 Rosario - Argentina \\ zandron@ifir-conicet.gov.ar
}

\begin{abstract}
The main features of the different linear gravity theories are reviewed. In particular, the supersymmetric extension of the JackiwTeitelboim $(1+1)$ linear gravity is considered in detail within the canonical exterior formalism. In this context the role of the several fields are analyzed. The constraints and the field equation are found. Finally, this supergravity model is treated in the second order formalism.
\end{abstract}

\section{Introduction}

The first model of two-dimensional gravity was proposed by Jackiw and Teitelboim (JT). It was obtained by dimensionally reducing the usual EinsteinHilbert action in $(2+1)$ dimensions $[1,2]$. Later, the string-inspired dilaton gravity was proposed $[3,4]$. From the gauge -theoretic formulation point of view, both models have the remarkable property of possessing a topological and gauge-invariant formulation $[5,6,7,8]$.

Frequently, linear $(1+1)$ - dimensional gravity or supergravities are used as a theoretical laboratory for studying properties also present in supergravities of greater dimensions. Lately, there has been a renewed interest in $(1+1)$ dimensional models. Perhaps one reason is the presence of black holes in this models $[3,4,9]$. Another reason is that, because of the simplicity of the $(1+$ 1)- dimensional models, one can gain a better understanding of the quantum

\footnotetext{
${ }^{1}$ Member of the Consejo Nacional de Investigaciones Científicas y Técnicas - Argentina
} 
treatment of gravity theories without the difficulties of the four-dimensional world.

In Refs. [10,11], following different approaches, the $\mathrm{N}=1$ supersymmetric extensions of the above-mentioned models were developed. In Ref. [10] the supersymmetric extension of the JT model is given in a superfield formalism, where after integrating out the Grassmann variables and eliminating the auxiliary fields by using their classical equations of motion, the supersymmetric action can be written in $(1+1)$ space-time. In the framework of gauge theory, this model is bases on the two-dimensional graded de Sitter group, whose associated graded algebra is the well known $\operatorname{osp}(1,1 \mid 1)$ one.

As it can be seen from Refs. $[10,12])$, the supersymmetric action in components can be written in terms of the field variables $V^{a}, \omega=\frac{1}{2} \varepsilon_{a b} \omega^{a b}$, and $\chi^{\alpha}$, i.e, the zweibein, the spin connection and the gravitino gauge fields respectively.

Interesting two-dimensional gravitational and vector gauge theories by reduction of $D=3$ topologically massive ones were also considered[13]. Subsequently, these research engendered much further ones[14].

Several years ago, a class of linear gravity theories based on the Riemann scalar curvature $R$ were proposed. The simplest of these models[1] requires an additional non-geometrical field $\phi$ in the action

$$
I_{1}=\int d^{2} x \phi(R-\lambda)
$$

where $\phi$ is an invariant world scalar acting as a Lagrangian multiplier enforcing the field equation

$$
R-\lambda=0 \text {. }
$$

Once the additional field has been introduced various generalizations and modifications of (1) and (2) can be considered, and so different dynamics for the fields $R$ and $\phi$ are possible.

Later on $[3,4]$, a similar model which is "string inspired" was given and whose action writes

$$
I_{2}=\int d^{2} x(\phi R-\lambda) .
$$

In this case the equation of motion from variying $\phi$ is

$$
R=0 \text {, }
$$

while variying the flat metric $g_{\mu \nu}=h_{\mu \nu}$ the equation reads

$$
\partial_{\mu} \partial_{\nu} \phi=\frac{1}{2} \lambda h_{\mu \nu}
$$


Consequently, the "black-hole" solution appears $[9,15]$, and the model becomes interesting from the quantum point of view.

In this paper we investigate the $N=1$ supersymmetric extension of the JT model in the second-order canonical formalism. Other two-dimensional supergravity theories, for instance the supersymmetric extension of the stringinspired dilaton gravity model, could also be treated in the same way.

The paper is organized as follows: In section 2, the main features of different two dimensional linear gravities are reviewed. The $\mathrm{N}=1$ supersymmetric extension of the model based on the graded de Sitter group is also analyzed. In section 3, starting from the or Canonical Exterior Formalism (CEF)the total Hamiltonian, the constraints and the equation of motion are found. In section 4 , the second-order formalism is constructed and the set of first-class constraints, generators of all the local gauge symmetries of the model, is computed by projecting on the spatial surface some of the motion equation obtained in the CEF. Finally, in section 5 the conclusions are given.

\section{Reviewing the main features of $(1+1)$-dimensional gravity and supergravity}

From the gauge-theoretic formulation point of view, the above models have the remarkable property of possessing a topological and gauge invariant formulation[5, $6,7,8]$.

Of particular interest are the gauge theoretical formulation given in Refs. [5,6]. To this end the de Sitter or anti-de Sitter groups are used satisfying the $S O(2,1)$ algebra

$$
\left[P_{a}, J\right]=\varepsilon_{a b} P^{b} \quad, \quad\left[P_{a}, P_{b}\right]=-\frac{1}{2} \lambda \varepsilon_{a b} J
$$

where $J$ and $P_{a}$ are respectively the Lorentz and translation generators.

The discussion about the problem of the gauge theoretical formulation for the action $I_{2}$ was given in Ref. [1] and it is based on the non-semisimple Poincaré group whose algebra

$$
\left[P_{a}, J\right]=\varepsilon_{a b} P^{b} \quad, \quad\left[P_{a}, P_{b}\right]=0
$$

is the $\lambda \rightarrow 0$ contraction of the algebra (6). However, there are various unexpected features in this formulation. For instance, it can be shown[8] that the transformation law for the Lagrange multipliers is an unfamiliar affine expression; the Lagrangian density is not invariant but changes by a total derivative. It is possible to construct an invariant Lagrangian density[8] by considering 
the following centrally extended Poincaré algebra, which is an unconventional contraction of the algebra (6)

$$
\left[P_{a}, J\right]=\varepsilon_{a b} P^{b} \quad, \quad\left[P_{a}, P_{b}\right]=\varepsilon_{a b} \frac{i}{2} \lambda I .
$$

In (8) was added the central element $I$ to the generators which produce a modification of the translation algebra. In Ref. [8], taking into account the new connection and curvature generated by the central element $I$, the gauge transformations and the invariance of the Lagrangian density of this model were discused.

We see that various model of linear gravity theories involving non-geometrical fields acting as Lagrange multipliers can be constructed. From the gauge invariant point of view two different members can be distinguished: The original model[1,2] based on the $S O(2,1)$ group and the "string-inspired" model[3, 4] based on the extended Poincaré group. In particular in Ref.[8] the model is based on an unconventional contraction of the $S O(2,1)$ model. This last is possible because of the ambiguity of two-dimensional angular momentum. Really, in Eqs.(6) it can be replaced $J$ by $J+s I / i$ and $\lambda$ by $\lambda / s$ and set $s \rightarrow \infty$ giving rise to the Eqs. (8).

Recently[16, 17], the two-dimensional reduction of the invariant action of the gravitational Chern-Simons model was studied. The gravitational threedimensional Chern-Simons term can be reduced by a Kaluza-Klein like ansatz, decomposing the three-dimensional metric into a two-dimensional metric, a $U(1)$ gauge field $A=A_{\mu} d x^{\mu}$ and a scalar field $\phi$. Later on, by means of conformal invariance it is possible to choose $\phi=1$. The action of the emergent linear gravity theory depend on the scalar two-dimensional curvature and on the Abelian dual field strength $F$. Therefore the model describe the twodimensional gravity interacting with the gauge field one-form $A$. Classical solutions have been constructed locally in Ref.[16], while in Ref.[17] the solutions are extended at global level, in order to construct the Carter-Penrose diagrams.

In Ref.[16] starting from the Chern-Simons terms for the matrix-valued gauge connections $A_{\mu}$, the gravitational Chern-Simons term can be constructed for the spin connection $\Omega_{\mu}$. Later on, by means of dimensional reduction it is shown how the three-dimensional gravitational Chern-Simons term produce a two-dimensional topological theory. The dimensional reduction is carried out by means of a Kaluza-Klein ansatz for the three-dimensional metric tensor. Apart from surface contribution the two-dimensional action writes

$$
I_{C S}=-\frac{1}{8 \pi^{2}} \int_{M_{2}} d^{2} x \sqrt{-g}\left(f r-f^{3}\right)
$$


where $r$ is the two-dimensional scalar curvature and moreover (9) depends on the Abelian dual field strength $\mathrm{f}=-2^{*} \mathrm{dA}$ (being $*$ the Hodge operation). Thus, a two-dimensional gravity theory interacting with the gauge field one-form $A$ appears.

So, in this context the equations of motion are found, and the local classical solutions are constructed. It is shown that two types of solutions exist, symmetry breaking and kink solutions, which structurally are similar to certain flat-space kinks. It is interesting to note that the kink make possible an space whose geometry is asymptotically anti-de Sitter. At small distances the scalar curvature is positive and it is vanishing at an intermediate point. So, the effect of the kink is analogous to a geometric gravitational force. The twodimensional action (9) is formally similar to the action of the dilaton model, when the field $f$ is identified with the dilaton field.

In Ref.[17] the discussion to a global level is given. This is done by written the action (9) using target space coordinates $Y, X, X^{+}, X^{-}$and gauge fields $A$, $\omega, V^{+}$and $V^{-}$. Therefore, the action (9) is equivalent to the following gravity first order action

$$
I_{C S}=\frac{1}{4 \pi^{2}} \int_{M_{2}} d^{2} x\left[X_{a}(D \wedge V)^{a}+X d \wedge \omega+Y d \wedge A+\varepsilon \Lambda(X, Y)\right]
$$

where $\Lambda(X, Y)=\frac{1}{2}\left(X Y-X^{3}\right)$. In fact the transition from (10) to (9) is very easy. In equation (10) the coordinate $Y$ acts as Lagrange multiplier for gauge curvature. Variation of $Y$ in (10) yields $X=f$, precisely the dual field strength in (9). Moreover, the first term in the action (10) requires vanishing torsion, and so the spin connection is replaced by its expression in a second order formalism. Applying well known methods used in the framework of first order gravity models, it is possible to construct solutions and to discuss their global properties and structure. It can be seen that the reformulation (10) shows advantages from classical as well as quantum point of view[14, 18].

In Refs. $[10,11,12]$, following different approaches, the $N=1$ supersymmetric extensions of the above mentioned models were developed. In Ref.[10] the starting point is the supersymmetric extension of the JT model whose action was written in Eq.(1). This is done by using a superfield formalism in superspace, that after integrating out the Grassmann variables and eliminating the auxiliary fields by using the classical equations of motion, the supersymmetric action can be written in $(1+1)$ spacetime. In the framework of gauge theory, this model is based on the two-dimensional graded de Sitter group, whose graded algebra associated to de Sitter supergroup $O s p(1,1 \mid 1)$ is given by 


$$
\begin{aligned}
& {\left[P_{a}, P_{b}\right]=-\frac{\lambda}{4} \varepsilon_{a b} J \quad, \quad\left[P_{a}, J\right]=\varepsilon_{a b} P_{b}} \\
& {\left[Q_{\alpha}, J\right]=\frac{1}{2}\left(\gamma^{5}\right)_{\alpha \beta} Q_{\beta}, \quad\left[P_{a}, Q_{\alpha}\right]=\frac{\lambda}{4}\left(\gamma_{a}\right)_{\alpha \beta} Q_{\beta}} \\
& \left\{Q_{\alpha}, Q_{\beta}\right\}=-2 i\left(\gamma^{a}\right)_{\alpha \beta} P_{a}+i \lambda\left(\gamma^{5}\right)_{\alpha \beta} J .
\end{aligned}
$$

Let $\mathcal{Q}_{\mathcal{A}}$ be any generator of the above graded algebra, where the compound index $A=[(a b), a, \alpha]$ runs in the tensor, the vector and the spinor ranges, respectively. Then, the graded invariant non-degenerate inner product, defined by $\left\langle\xi^{A} \mathcal{Q}_{A}, \eta^{B} \mathcal{Q}_{B}\right\rangle=(-1)^{|A||B|} \xi^{A} \eta^{B}\left\langle\mathcal{Q}_{\mathcal{A}}, \mathcal{Q}_{\mathcal{B}}\right\rangle$, is explicitly written as

$$
\left\langle P_{a}, P_{b}\right\rangle \equiv h_{a b} \quad, \quad\langle J, J\rangle \equiv \frac{4}{\lambda^{2}} \quad, \quad\left\langle Q_{\alpha}, Q_{\beta}\right\rangle \equiv-\frac{8 i}{\lambda} \varepsilon_{\alpha \beta},
$$

where $|A|$ and $|B|$ indicate the Fermi grading.

Consequently, the action for the supersymmetric extension of the JT model is formally written as

$$
S=\int d^{2} x \varepsilon^{\mu \nu}\left\langle\zeta, F_{\mu \nu}\right\rangle,
$$

where $F=d \mu+\mu \wedge \mu$ is the field strength associated with the algebra-valued one-form gauge field

$$
\mu=V^{a} P_{a}-\omega J+\frac{1}{2} \xi^{\alpha} \gamma^{5} Q_{\alpha}
$$

In Eq.(14) $V^{a}, \omega=\frac{1}{2} \varepsilon_{a b} \omega^{a b}$ and $\xi^{\alpha}$ are, respectively, the zweibein, the spin connection and the gravitino gauge fields; and $\zeta=\eta^{a} P_{a}+\eta^{J} J+\eta^{\alpha} Q_{\alpha}$ is a world scalar with value in the graded algebra (11).

Therefore, the explicit form of the supersymmetric action (13) in components is written as follows

$$
\begin{aligned}
S & =\int d^{2} x(-g)^{1 / 2}\left[\eta^{a} \varepsilon^{\mu \nu}\left(\partial_{\mu} V_{a \nu}-\varepsilon_{a b} \omega_{\mu} V_{\nu}^{b}-\frac{i}{4} \bar{\xi}_{\mu} \gamma_{a} \xi_{\nu}\right)\right. \\
& -\frac{4}{\lambda^{2}} \eta^{J} \varepsilon^{\mu \nu}\left(\partial_{\mu} \omega_{\nu}+\frac{1}{8} \lambda^{2} \varepsilon^{a b} V_{a \mu} V_{b \nu}+\frac{i}{8} \lambda \bar{\xi}_{\mu} \gamma_{5} \xi_{\nu}\right) \\
& \left.+\frac{4 i}{\lambda}\left(\varepsilon^{\mu \nu} D_{\mu} \bar{\xi}_{\nu} \gamma_{5}+\frac{1}{4} \lambda \bar{\xi}_{\mu} \gamma_{\mu}\right) \eta\right]
\end{aligned}
$$

where we choose the $2 \times 2$ real $\gamma$-matrices satisfying $\gamma_{a} \gamma_{b}=\eta_{a b}+\gamma^{5} \varepsilon_{a b}, \gamma^{5}=$ $\gamma^{0} \gamma^{1}$

In Ref. [11], an $\mathrm{N}=1$ supersymmetric version of two-dimensional gravity coupled to matter is analyzed. At least two interesting results are clearly 
found: i) the supersymmetry is used to prove positive-energy theorems for a large class of generalized dilaton gravity; ii) supersymmetry suggests a spinorial expression for the Arnowitt-Deser-Misner energy $M$. By using this expression is proven that $M$ is non-negative for smooth initial data asymptotic to the linear dilaton vacuum.

Recently[19], the Bogomolnyi-Prasad-Sommerfield black holes were studied in the framework of the two-dimensional dilaton supergravity. This was done in the first order formalism by starting from the action for the graded Poisson-Sigma model. The possible solutions with vanishing and non-vanishing fermions were found. The knowledge of the general analytic solution in twodimensional dilaton supergravity plays an important role. In particular it is shown that the geometry of solutions with non-vanishing fermions must be Minkowski space and so, there does not exist supersymmetric black holes with dilatino or gravitino.

Therefore, the interesting results obtained from the supersymmetric extension of two dimensional linear gravities, gives a reason to found these models by using the supergroup manifold approach.

\section{Canonical exterior formalism for a $(1+1)$-dimensional supergravity model}

Let us consider the problem by starting from the first-order (CEF) on group manifold $[20,21,22]$. So, the first step is to write the Lagrangian density defined by (15) using exterior calculus. For a supergroup $G$ and a bosonic gauge subgroup $H \subset G$, the physical superspace is defined by the coset manifold $M^{2}=G / H$. The Lagrangian density is a bosonic 2-form functional of the dynamical fields and their exterior derivatives. Because the physical content of the theory is present in the coset manifold $M^{2}$, all the fields must be considered only as reduced forms, i.e. forms defined on $M^{2}$. Moreover, these forms are written in the holonomic basis $d x^{\mu}(\mu=0,1)$.

In the first-order exterior formalism the dynamics is described by the three 1-form gauge fields $\omega^{a b}$ (spin connection), $V^{a}$ (zweibein) and $\xi$ (gravitino) and the three 0 -form fields $\eta^{J}, \eta_{a}$ and $\eta$. The three 0 -form fields are non-geometrical objects and are introduced with the purpose of obtaining rheonomic equations of motion, i.e. equations compatible with the Bianchi identities as it is required by the group manifold approach.

Consequently, the Lagrangian density is written as

$$
\mathcal{L}=\eta_{a} R^{a}-\frac{4}{\lambda^{2}} \eta^{J} R+\frac{4 i}{\lambda} \bar{\rho} \gamma^{5} \eta-\frac{1}{2} \eta^{J} V^{a} V^{b} \varepsilon_{a b}
$$




$$
-\frac{i}{2 \lambda} \eta^{J} \bar{\xi} \gamma^{5} \wedge \xi-i\left(\bar{\xi} \gamma^{b} \eta\right) \wedge V^{a} \varepsilon_{a b}
$$

In (16) $\mathrm{R}$ (Riemann curvature), $R^{a}$ (torsion) and $\rho$ (gravitino curvature) are the curvature 2-forms corresponding to the gauge fields and they are given by

$$
\begin{gathered}
R=d \omega \\
R^{a}=\mathcal{D} V^{a}-\frac{i}{4} \bar{\xi} \gamma^{a} \wedge \xi=d V^{a}-\omega \wedge V_{b} \varepsilon^{a b}-\frac{i}{4} \bar{\xi} \gamma^{a} \wedge \xi \\
\bar{\rho}=\mathcal{D} \bar{\xi}=d \bar{\xi}+\frac{1}{2} \omega \wedge \bar{\xi} \gamma^{5} .
\end{gathered}
$$

Looking at the Lagrangian density (16) we see that it is linear in the curvatures taking the general form $\mathcal{L}=R^{A}(\mu) \wedge \nu_{A}(\mu)+\Lambda(\mu)$. In the group manifold approach the third principle states that the functional coefficients $\Lambda(\mu)$ and $\nu_{A}(\mu)$ must satisfy the condition [23]

$$
A\rfloor \Lambda+\mathcal{D} \nu_{A}=0,
$$

for the vacuum solution $R^{A}=0$. The coefficients $\Lambda(\mu)$ and $\nu_{A}(\mu)$ do not depend on the spin connection and they must be invariant under transformation of the bosonic gauge symmetry group $H$.

The idea is to construct the first-order CEF starting form a Lagrangian that differs from (16) by an exterior derivative i.e, a Lagrangian density not containing spin-connection derivatives. This will be useful in the second-order formalism where the canonical conjugate momentum to the spin connection field variable $\omega$ is a strongly equal to zero quantity.

As it is well known in the CEF the canonical conjugate momenta $\pi_{A}=$ $\delta \mathcal{L} / \delta d \mu^{A}$ of the fields $\mu^{A}=\left(\omega, V^{a}, \xi, \eta^{J}, \eta_{a}, \eta\right)$ are obtained by functional variation of the Lagrangian with respect to the "velocities" $d \mu^{A}$. So, the momenta associated with the three gauge fields are 0 -forms and the others three momenta associated with the auxiliary fields are 1-forms.

Therefore, by looking at the Lagrangian (16) we see that the set of primary constraints are written

$$
\begin{gathered}
\Phi_{a}(V)=\pi_{a}(V)-\eta_{a} \approx 0, \\
\Phi_{a}(\omega)=\pi(\omega) \approx 0,
\end{gathered}
$$




$$
\begin{gathered}
\Phi_{\alpha}(\bar{\xi})=\pi_{\alpha}(\bar{\xi})-\frac{4 i}{\lambda}\left(\gamma^{5} \eta\right)_{\alpha} \approx 0, \\
\Phi^{a}\left(\eta_{a}\right)=\pi^{a}\left(\eta_{a}\right) \approx 0, \\
\Phi_{J}\left(\eta^{J}\right)=\pi_{J}\left(\eta^{J}\right)-\frac{4}{\lambda^{2}} \omega \approx 0 \\
\bar{\Phi}^{\alpha}\left(\eta_{\alpha}\right)=\bar{\pi}^{\alpha}\left(\eta_{\alpha}\right) \approx 0 .
\end{gathered}
$$

By considering the definition and properties of the graded form-brackets $[21]$, it is possible to compute the form-brackets $\left(\Phi_{A}, \Phi_{B}\right)$ for pairs of constraints. It is straigthforward to prove that all the primary constraints (21)(26) are second-class ones, that is

$$
\left(\Phi_{A}, \Phi_{B}\right) \neq 0
$$

In the $\mathrm{CEF}$, the conserved first-class dynamical quantity describing the dynamics of the system is the extended Hamiltonian $H_{T}$, and it is the bosonic two-form defined by [21]

$$
H_{T}=H_{c a n}+\Lambda^{A} \wedge \Phi_{A}
$$

where the Lagrange multipliers $\Lambda^{A}$ can be unambiguously determined. This is done when the fundamental equation of motion in the CEF is taken into account giving rise to the general result

$$
\Lambda^{A}=d \mu^{A}
$$

In (28) the canonical Hamiltonian $H_{c a n}=d \mu^{A} \wedge \pi_{A}-\mathcal{L}$ is given by

$$
\begin{aligned}
H_{c a n} & =\eta_{a}\left[\omega \wedge V_{b} \varepsilon^{a b}+\frac{i}{4}\left(\bar{\xi} \wedge \gamma^{a} \xi\right)\right]+\eta^{J}\left[\frac{1}{2} V^{a} \wedge V^{b} \varepsilon_{a b}\right. \\
& \left.+\frac{i}{2 \lambda}\left(\bar{\xi} \wedge \gamma^{5} \xi\right)\right]+\left[-\frac{2 i}{\lambda} \omega \wedge \bar{\xi}+i\left(\bar{\xi} \gamma^{b}\right) \wedge V^{a} \varepsilon_{a b}\right] \eta .
\end{aligned}
$$

The field equations of motion in the CEF are given by the consistency conditions on the primary constraints, i.e, $d \Phi_{A}=\left(\Phi_{A}, H_{T}\right) \approx 0$. After some algebraic manipulation they read

$$
d \Phi_{a}(V)=\left[\eta_{b} \omega \varepsilon_{a b}+\eta^{J} V^{b} \varepsilon_{a b}-i\left(\bar{\xi} \gamma^{b} \eta\right) \varepsilon_{a b}-d \eta_{a}\right] \approx 0
$$




$$
\begin{gathered}
d \Phi(\omega)=\left[\eta_{a} V_{b} \varepsilon^{a b}-\frac{2 i}{\lambda}(\bar{\xi} \eta)+\frac{4}{\lambda^{2}} d \eta^{J}\right] \approx 0 \\
d \Phi(\bar{\xi})=\left[\frac{i}{2} \eta_{a} \gamma^{a} \xi+\frac{i}{\lambda} \eta^{J} \gamma^{5} \xi+\frac{2 i}{\lambda} \omega \eta+i \gamma^{b} \eta V^{a} \varepsilon_{a b}-\frac{4 i}{\lambda} \gamma^{5} d \eta\right] \approx 0 \\
d \Phi^{a}\left(\eta_{a}\right)=-\left[\omega \wedge V_{b} \varepsilon^{a b}+\frac{i}{4} \bar{\xi} \gamma^{a} \wedge \xi-d V^{a}\right] \approx 0 \\
d \Phi_{J}\left(\eta^{J}\right)=-\left[\frac{1}{2} V^{a} \wedge V^{b} \varepsilon_{a b}+\frac{i}{2 \lambda}\left(\bar{\xi} \gamma^{5} \wedge \xi\right)+\frac{4}{\lambda^{2}} d \omega\right] \approx 0 \\
d \bar{\Phi}(\eta)=-\left[\frac{2 i}{\lambda} \omega \wedge \bar{\xi}-i \bar{\xi} \gamma^{b} \wedge V^{a} \varepsilon_{a b}+\frac{4 i}{\lambda} d \bar{\xi} \gamma^{5}\right] \approx 0
\end{gathered}
$$

The Lagrangian density (16) is rheonomic, i.e. the solution for the curvatures $\left(R^{a}, R, \rho\right)$ associated to the gauge fields must be compatible with the Bianchi identities. Moreover, the parametrization of these curvatures are obtained directly from the 2-form field equations (31)-(33) and they may be written as follows

$$
\begin{gathered}
R^{a}=0 \\
R=-\frac{1}{8} \lambda^{2} V^{a} \wedge V^{b} \varepsilon_{a b}-\frac{i}{8} \lambda \bar{\xi} \gamma^{5} \wedge \xi \\
\bar{\rho}=\frac{1}{4} \lambda \bar{\xi} \gamma^{b} \gamma^{5} \wedge V^{a} \varepsilon_{a b} .
\end{gathered}
$$

In fact from the above equations it can be seen that the inner-outer $(V \wedge \xi)$ and the outer-outer $(\xi \wedge \xi)$ components of the curvatures remain determined in terms of the inner-inner $(V \wedge V)$ component. In this case the inner-inner component of $R$ is proportional to the square of the cosmological constant $\lambda$.

On the other hand we can see how the auxiliary 0 -form fields $\eta^{J}, \eta_{a}$ and $\eta$ can be interpreted from the CEF. For these non-geometrical fields, the curvatures are substituted by the covariant exterior derivatives. Thus, the 1-form field equations (34)-(36) are also rheonomic and they are written in inner and outer components as follows

$$
\begin{gathered}
\mathcal{D} \eta_{a}=d \eta_{a}-\omega \varepsilon_{a b} \eta^{b}=\eta^{J} V^{b} \varepsilon_{a b}+i \varepsilon_{a b} \bar{\eta} \gamma^{b} \xi \\
\mathcal{D} \eta^{J}=d \eta^{J}=-\frac{1}{4} \lambda^{2} \eta_{a} \varepsilon^{a b} V_{b}+\frac{i}{2} \lambda \bar{\eta} \xi
\end{gathered}
$$




$$
\mathcal{D} \eta=d \eta-\frac{1}{2} \omega \eta \gamma^{5}=\frac{1}{4} \lambda \varepsilon_{a b}\left(\gamma^{b} \gamma^{5} \eta\right) V^{a}+\frac{1}{4}\left(\eta^{J}+\frac{1}{2} \lambda \eta_{a} \gamma^{a} \gamma^{5}\right) \xi
$$

From the above construction it can be seen that the CEF is covariant in all the steps. However, it is not a proper Hamiltonian formalism because the extended Hamiltonian $H_{T}$ is not a true generator of time evolutions and the form-brackets do not contain the same information as the Poisson brackets. The CEF can be related with the Hamiltonian formalism in components, and so the form-brackets can be related to the Poisson brackets but not in a trivial way[20]. The integral relationship which relates the form-brackets $(A, B)$ to the Poisson brackets between forms $[A(x), B(y)]$ is given by $[20,21]$

$$
(-1)^{a+1} \int_{\Sigma} \alpha \wedge(A, B) \wedge \beta=\iint_{\Sigma \times \Sigma} \alpha(x) \wedge[A(x), B(y)] \wedge \beta(y)
$$

where $a$ is the degree of the form $A$ and $\alpha, \beta$ are text forms.

Once the space time decomposition is done and the surface $\Sigma$ remains defined, the ordinary Poisson brackets are obtained by expanding the forms $A(x)$ and $B(y)$ in the holonomic bases $d x^{i}, d y^{j}$ and then the ordinary Poisson brackets between fields and momenta components can be used.

Before to conclude this section a further consideration about the exterior canonical formalism must be done: as it was said, all the primary constraints provided by the CEF are second-class ones, and so they are not related with the gauge symmetry of the model. Moreover, the possibility of using different Lagrangian densities means that there is not a unique set of canonical conjugate momenta and consequently there is not a unique set of primary constraints in the CEF. On the other hand, in the second-order formalism the second-class constraints must be eliminated. As it is well known this is done by defining the Dirac brackets from the Poisson brackets. As it is well known the Dirac brackets $[F, G]^{D}$ for generic functional $F$ and $G$ are obtained from the set of second-class constraints $\Psi_{A}$ by means of the definition

$$
[F, G]^{D}=[F, G]-\left[F, \Psi_{A}\right] C^{A B}\left[\Psi_{B}, G\right]
$$

where $C^{A B}\left[\Psi_{B}, \Psi_{C}\right]=\delta_{C}^{A}$ for the compound indices $A, B, C$. To compute the Dirac brackets (44) we must consider the restriction to $\Sigma$ of all the second-class constraints (21)-(26) i.e, $\Psi_{A}=\left.\Phi_{A}\right|_{\Sigma}$.

As it is well known, the main properties of the Dirac brackets are:

i) If one of the function $F$ or $G$ is first class, then

$$
[F, G]^{D} \approx[F, G]
$$


In particular for the Hamiltonian $\mathcal{H}$ holds

$$
[F, \mathcal{H}]^{D} \approx[F, \mathcal{H}]
$$

This means that the same equations of motion are obtained by using the Poisson or the Dirac brackets. Thus, the rate of change in time of any functional $F$ of the canonical variables is also given by

$$
\dot{F}=[F, \mathcal{H}]^{D}
$$

ii) For any functional $F$ of the canonical variables it is

$$
\left[\Psi_{A}, \mathcal{H}\right]^{D}=0
$$

Therefore we can set $\Psi_{A}=0$ either before or after evaluating the Dirac brackets.

As it is well known, once the Dirac brackets are evaluated from equation (44), the transition to quantum theory is realized as usual in a canonical formalism by replacing classical fields by quantum field operators acting on some Hilbert space.

\section{Second-order formalism and constraints.}

When the model is considered from the quantum point of view, the secondorder formalism is necessary. It is in this formalism where the dynamical degrees of freedom are separated from those of gauge degrees of freedom.

With this aim and in order to find the constraints in the second-order formalism, the first step is to make the spacetime decomposition in the manifold $M^{2}$. Of course, when a privileged time direction is chosen in the manifold $M^{2}$, the manifest covariance is lost.

The notation and conventions are: tangent space indices are denoted by $a, b=1,2$, space-time indices by $\mu, \nu=0,1$, space indices $i, j=1 ; \quad \eta_{a b}=$ $(+-) ; \varepsilon_{a b}=V_{a \mu} V_{b \nu} \varepsilon^{\mu \nu} ; g_{\mu \nu}^{(2)}=V_{a \mu} V_{\nu}^{a}$. In the space-time decomposition it is convenient to introduce the shift and lapse functions $N^{i}$ and $N^{\perp}$, which determine the components of the metric tensor. The zweibein 1-form is written $V_{a}=V_{a \mu} d x^{\mu}$, where the holonomic components are $V_{a \mu}=\left(V_{a i}^{(2)}, V_{a 0}\right)$, and

$$
V_{a i}^{(2)}=V_{a i}^{(1)}=V_{a i}, \quad V_{a}^{(1) i}=V_{a}^{i}, \quad V_{a}^{(2) i}=V_{a}^{(1) i}+\left(N^{\perp}\right)^{-1} N^{i} n_{a}
$$

The normal $n_{a}$ satisfies $n_{a} n^{a}=-1, n_{a} V_{i}^{a}=0, n_{a}=-N^{\perp} V_{a}^{0}$ and $\left(-g^{(2)}\right)^{1 / 2}=N^{\perp} g^{1 / 2}$ with $g=\operatorname{det}\left(g^{(1)}\right)$. 
An arbitrary vector $\mathcal{V}^{a}$ can be decomposed as $\mathcal{V}^{a}=\mathcal{V}^{\perp} n^{a}+\mathcal{V}^{i} V_{i}^{a}$, with $\mathcal{V}^{\perp}=-\mathcal{V}_{\perp}=-n_{a} \mathcal{V}^{a}$ and $\mathcal{V}_{i}=\mathcal{V}^{a} V_{a i}$

The time variable is chosen so that the 1 -form $d x^{0}$ can be detached. More precisely, we consider fields and forms defined on a spacelike $x^{0}=t=t^{0}$ onedimensional "surface" $\Sigma$, by considering the injection map $\chi: \Sigma \rightarrow M^{2}$. Thus, the associated pullback $\chi^{*}$ acts on any form by setting $t=t^{0}$ and $d t^{0}=0$.

In order to obtain the final form of the generator of time evolutions in the canonical component formalism, the metricity condition in one and two dimensions must be considered. The general equation relating the two-dimensional spin connection $\omega^{a b}$ with the one-dimensional spin connection $\Omega^{a b}$ writes

$$
\omega_{i}^{a b}=\Omega_{i}^{a b}+\left(n^{b} V^{a j}-n^{a} V^{b j}\right) K_{i j}
$$

where the extrinsic curvature $K_{i j}$ was introduced.

Similarly, from the metricity condition in one dimension, the following equation holds

$$
\partial_{i} n^{a}+\Omega_{i}^{a b} n_{b}=0 .
$$

The second-order formalism is obtained by solving the torsion field equation, given the following results for the spin connection

$$
\omega_{\mu}(V, \xi)=\omega_{\mu}(V)+\kappa_{\mu}(\xi)
$$

where $\omega_{\mu}(V)$ is given by

$$
\omega_{\mu}(V)=-\varepsilon^{\nu \rho} V_{\mu}^{a} \partial_{\nu} V_{a \rho},
$$

and the contorsion tensor $\kappa_{\mu}(\xi)$ is

$$
\kappa_{\mu}(\xi)=\frac{i}{4} \xi_{\mu} \gamma^{5} \gamma^{\nu} \xi_{\nu}
$$

All the quantities provided by the CEF i.e, total Hamiltonian, constraints and field equations must be projected on the "surface" $\Sigma$. Once the canonical conjugate momenta $\pi_{A}$ are written in terms of the spatial components $d x^{i}$ of the holonomic basis, the Poisson brackets between pairs of canonical variables remain defined as usual.

Another question to take into account is that the CEF plays, with respect to the first order canonical component formalism, an analogous role to that played by the first order canonical component formalism with respect to the second order one. Therefore, we will consider that all the primary constraints in the CEF remain at least weakly zero in the canonical component formalism (see, for instance Refs.[20,22]). 
On the other hand, we also assume that the restrictions to $\Sigma$ of the constraints (22)-(26) are strongly equal to zero i.e, $\chi^{*} \Phi_{A}=0$. For the remaining constraint $\Phi_{a}(V)$ the restriction to $\Sigma$ is maintained as a weakly zero quantity

$$
\chi^{*} \Phi_{a}=\Psi_{a} \approx 0 .
$$

The bosonic 2-form (28) provides by the CEF can be written as follows

$$
\int H_{T}=\int d x^{0} \tilde{\mathcal{H}}
$$

where the time variable is chosen so that the 1 -form $d x^{0}$ can be detached. The remaining bosonic one-form integrated in one dimension is the proper Hamiltonian generator of time evolutions and it turns out to be of the form

$$
\tilde{\mathcal{H}}=\int d x\left(\frac{1}{2} \omega_{0}^{a b} \mathcal{H}_{a b}+V_{a 0} \mathcal{H}^{a}+\bar{\xi}_{0 \alpha} \mathcal{H}^{\alpha}\right)
$$

where

$$
\begin{gathered}
\mathcal{H}_{a b} d x=\left.\left(\Phi_{a} V_{b}-\Phi_{b} V_{a}\right)\right|_{\Sigma} \approx 0 \\
\mathcal{H}^{a} d x=\left.\left[-d \eta^{a}+\eta^{J} V_{b} \varepsilon^{a b}+\omega \varepsilon \eta_{b}-i\left(\bar{\xi} \gamma_{b} \eta\right) \varepsilon^{a b}+\omega \varepsilon^{a b} \Phi_{b}\right]\right|_{\Sigma} \approx 0 \\
\mathcal{H}^{\alpha} d x=\left[-\frac{4 i}{\lambda}\left(\gamma^{5} d \eta\right)^{\alpha}+\frac{i}{2} \eta_{a}\left(\gamma^{a} \xi\right)^{\alpha}+\frac{i}{\lambda} \eta^{J}\left(\gamma^{5} \xi\right)^{\alpha}+\frac{2 i}{\lambda} \omega \eta\right. \\
\left.+i\left(\gamma^{b} \eta\right)^{\alpha} V^{a} \varepsilon_{a b}+\frac{i}{2}\left(\gamma^{a} \xi\right)^{\alpha} \Phi_{a}\right]\left.\right|_{\Sigma} \approx 0 .
\end{gathered}
$$

In Eqs.(57) and (60) the spinor index $\alpha$ was explicitly written.

Subsequently, by using Eqs. (23) and (25) the spinor field $\eta$ and the spin connection $\omega$ must be eliminated from the Eqs. (59) and (60). Moreover, the boson field $\eta_{a}$ is eliminated by means of (32). In order to arrive at Eqs.(58)(60), the above prescriptions about the role of the constraints in the CEF have been taken into account.

Consequently, from (58) we can see that the antisymmetric weakly zero quantity $\mathcal{H}_{a b}=J_{a b}$ is the generator of local Lorentz rotations, that in this context naturally appears when the space-time decomposition is carried out. Contrarily, in the JT component formalism the generator of local Lorentz rotations $J=\frac{1}{2} \varepsilon^{a b} J_{a b}$ must be introduced $a d$ hoc by demanding the closure of the constraint algebra. 
By considering (21) and (55) the constraint $\Psi_{a}$ can be computed explicitly, and after some algebraic manipulation it is possible to write the following equation for the 0 -form $\mathcal{H}_{a b}$

$\mathcal{H}_{a b}=\Psi_{a}^{i} V_{b i}-\Psi_{b}^{i} V_{a i}=\Pi_{a}^{i} V_{b i}-\Pi_{b}^{i} V_{a i}+\frac{4}{\lambda^{2}} g^{1 / 2}\left(n_{a} V_{b}^{i}-n_{b} V_{a}^{i}\right)\left(\partial_{i} \eta^{J}-\frac{i}{2} \lambda \bar{\xi}_{i} \eta\right)$

where $\Pi_{a}^{i}=\left.\pi_{a}^{i}\right|_{\Sigma}$, being $\pi_{a}^{i}$ the componets of the conjugate momentum of the zweibein field. In (61) we call $\Psi_{a}^{i}=g^{1 / 2} \varepsilon^{0 i} N^{\perp} \Psi_{a}$, that after some algebra it can be written as

$$
\Psi_{a}^{j}=V_{a i} \pi^{i j}-g^{1 / 2} \varepsilon^{0 j} N^{\perp} \eta_{a}-n_{a} J^{j \perp}-\frac{4}{\lambda^{2}} n_{a} g^{1 / 2} g^{i j}\left(\partial_{i} \eta^{J}-\frac{i}{2} \lambda \bar{\xi}_{i} \eta\right) .
$$

Analogously, the other two 0 -form $\mathcal{H}^{a}$ and $\mathcal{H}^{\alpha}$ are first-class contraints and they are written

$$
\begin{aligned}
\mathcal{H}^{a} & =-n^{a}\left[-\pi_{i}^{i} K_{k}^{k}+g^{1 / 2} \eta^{J}-\frac{4}{\lambda^{2}} g^{1 / 2} g^{j k} \nabla_{j} \partial_{k} \eta^{J}-\frac{\lambda}{4} \bar{\xi}_{j} \gamma_{\perp} \pi^{j}\right. \\
& \left.-\frac{1}{2} \partial_{i}\left(\bar{\xi}^{i} \gamma_{\perp} \gamma_{j} \pi^{j}\right)-\partial_{i} J^{i \perp}\right] \\
& +V^{a i}\left[-\frac{4}{\lambda^{2}} g^{1 / 2}\left(\partial_{i} \eta^{J}\right) K_{k}^{k}-\partial_{i} \pi_{k}^{k}+g^{-1 / 2} \partial_{i} g^{1 / 2} \pi_{k}^{k}-\frac{1}{4} \lambda \bar{\xi}_{i} \gamma_{j} \pi^{j}\right. \\
& \left.+-\frac{1}{2} K_{k}^{k}\left(\bar{\xi}^{i} \gamma_{\perp} \gamma_{j} \pi^{j}\right)+J_{i \perp} K_{k}^{k}\right], \\
\mathcal{H}^{\alpha} & =-\gamma^{5} \partial_{i} \pi^{i \alpha}+\frac{i}{\lambda} g^{1 / 2} \eta^{J}\left(\gamma_{\perp} \gamma^{i} \xi_{i}\right)^{\alpha}-\frac{\lambda}{4}\left(\gamma_{i} \gamma^{5} \pi^{i}\right)^{\alpha}+\frac{i}{2} \pi_{k}^{k}\left(\gamma^{i} \xi_{i}\right)^{\alpha} \\
& -\frac{2 i}{\lambda^{2}}\left(\gamma_{\perp} \xi^{j}\right)^{\alpha} \partial_{j} \eta^{J}-i g^{-1 / 2}\left(\bar{\xi}^{i} \gamma_{\perp} \gamma_{j} \pi^{j} \gamma_{\perp} \xi_{i}\right)^{\alpha} \\
& -\frac{\lambda^{2}}{8} g^{-1 / 2} \pi^{J}\left(\gamma_{\perp} \gamma_{i} \gamma^{5} \pi^{i}\right)^{\alpha}-\frac{i}{2}\left(\gamma_{\perp} \xi_{i}\right)^{\alpha} J^{i \perp}
\end{aligned}
$$

In the Eqs. (63) and (64) the canonical conjugate momenta corresponding to the bosonic fields $g_{i j}$ and $\eta_{J}$ and the fermionic field $\xi_{i \alpha}$ were introduced, and they respectively read

$$
\begin{gathered}
\pi^{i j}=-g^{1 / 2}\left(K^{(i j)}-g^{i j} K_{k}^{k}\right), \\
\pi_{J}=\frac{4}{\lambda^{2}} N^{\perp} \varepsilon^{o i} \omega_{i}(V, \xi)
\end{gathered}
$$




$$
\pi^{i \alpha}=\frac{4 i}{\lambda} N^{\perp} \varepsilon^{o i}\left(\gamma^{5} \eta\right)^{\alpha},
$$

where $K^{(i j)}$ is the symmetric part of the extrinsic curvature, and $K_{k}^{k}$ its trace.

As it is usual in the literature, by using the shift and lapse functions $N^{i}$ and $N^{\perp}$ already introduced, the first-class constraint $\mathcal{H}^{a}$ is decomposed as follows

$$
V_{a 0} \mathcal{H}^{a}=N^{\perp} \mathcal{H}_{\perp}+N^{i} \mathcal{H}_{i},
$$

where the components $\mathcal{H}_{\perp}$ and $\mathcal{H}_{i}$ are both first-class constraints.

Finally, it can be proven that the set of constraints $\mathcal{H}^{A}=\left(\mathcal{H}_{a b}, \mathcal{H}_{\perp}, \mathcal{H}_{i}, \mathcal{H}_{\alpha}\right)$ close the superalgebra

$$
\left[\mathcal{H}_{A}(x), \mathcal{H}_{B}(y)\right]=\Lambda_{A B}^{C} \mathcal{H}_{C}(x) \delta(x-y),
$$

where $\Lambda_{A B}^{C}=R_{A B}^{C}-C_{A B}^{C}$ are the structure functions for curvatures $R_{A B}^{C}$ and structure constant $C_{A B}^{C}$ of the graded Lie algebra. The Eq.(69) constitutes the so called constraint superalgebra.

\section{Conclusions}

The supersymmetric extension of the $(1+1)$ - dimensional Jackiw-Teiteiboim model was considered in the framework of the CEF. The dynamics of this constrained system found by applying the geometrical prescriptions of the exterior canonical formalism for the supergroup manifold.

As it was shown, the CEF is not a proper canonical formalism because it does not propagate data defined on an initial surface as it is required by a standard mechanical system.

In spite of this, at classical level, the CEF is a powerful method to understand the structure of the gravitational field. The CEF is covariant in all its steps because of the use of exterior algebra. This allows to find the equations of motion and the constraints in a very simple way without introducing complicate calculations. All the primary constraints are second-class ones.

The relation between the CEF and the usual first-order canonical formalism written in components was also given. This relation was done by means of a non trivial integral relationship between the form-brackets and the usual Poisson brackets.

As it was shown, the torsion equation allows to obtain the second-order canonical formalism starting from the first-order one.

In the Riemannian gravity case, the torsion equation $R^{a}=0$ must be considered as an strongly equal to zero constraint, and so the spin connection is solved in terms of the zweibein and the spinor field. 
In order to go over the second-order formalism, the space-time decomposition in $M^{2}$ was performed, losing the explicit covariance of all the equations. Once this is done, the Hamiltonian system is treated as usual according to the Dirac prescriptions. From the total Hamiltonian coming from the CEF is evaluated the proper Hamiltonian (57) as generator of time evolution. As it was shown the primary constraint $\Psi_{a}=\chi^{*} \Phi_{a}$ obtained in the CEF plays an important role in the construction of the first-class constraints. Finally, the proper Hamiltonian (57) is given in terms of the first-class constraints which close the constraint algebra. Therefore, all the Hamiltonian gauge symmetries remain determined and the apparent gauge degrees of freedom can be unambiguously removed leaving only the physical ones. When the model is considered from the quantum point of view this last step is necessary.

Therefore, we conclude that the CEF can be used as an interesting formal resource for deriving constraints and equations of motion due to their intrinsic geometrical language.

\section{References}

[1] Teitelboim C. 1983 Phys. Lett 126B 41, 46, 49.

[2] Jackiw R., Teitelboim C., in S. Christensen (Ed), Quantum Theory of Gravity, Adam Hilger, Bristol, UK, 1984.

[3] Verlinde H. 1992 6th Marcel Grossman Meeting on General Relativity ed H. Sato (Singapore: World Scientific)

[4] Callan C. Giddings S, Harvey A and Strominger A, 1992 Phys. Rev. D45 1005 .

[5] Fukuyama T. and Kamimura K., 1985 Phys. Lett 160B 259.

[6] Chamseddine A.H. and Wyler D, 1989 Phys. Lett 228B 75.

[7] Montano D. and Sonnenschein J, 1989 Nuclear Physics B313 258.

[8] Cangemi D. and Jackiw R, 1992 Phys. Rev. Lett 69233.

[9] Mann R.B. and Steele T.G 1992 Classical Quantum Grav. 9475.

[10] Chamseddine A. H 1991 Phys. Lett 258B 97.

[11] Park Y. and Strominger A. 1993 Phys. Rev. D47.

[12] Cangemi D Leblanc M, 1994 Nuclear Physics B420 363. 
[13] Aragone C., Deser S. and Ferrara S. 1987 Class. Q. Grav. 41003.

[14] Grumiller D., Kummer W. and Vassilevich D.V 2002 Phys. Rep. 369 327-429.

[15] Elitzur S., Forge A. and Rabinovici E. 1991 Nucl. Phys. B359 581; Witten E. 1991 Phys. Rev. D44 314.

[16] Guralnik G., Iorio A., Jackiw R. and Pi S.-Y. 2003 Ann. Phys. (NY) 308 222.

[17] Grumiller D. and Kummer W. 2003 Ann. Phys. (NY) 308211.

[18] Cattaneo A.S. and Felder G. 2000 Commun. Math. Phys. 212 591-611.

[19] Bergamin L, Grumiller D. and Kummer W. 2004 Journal of Physics A37 3881 and bibliography cuoted therein.

[20] Nelson J. and Regge T. 1986 Ann. Phys. (NY) 166234.

[21] Foussats A. and Zandron O.S. 1990 Int. J. Mod. Phys. A5 725.

[22] Foussats A. and Zandron O.S. 1990 Ann. Phys. (NY) 203207.

[23] Castellani L., D'Auria R. and Fré P. in Supersymmetry and Supergravity 1983, Proceeding 19th Winter School and Workshop of Theoretical Physics, Karpacz, Poland, ed. B. Milewski (World Scientific, 1983).

Received: April, 2010 